\title{
Education to Increase Skills in Research Methods among Clinicians in Health Care
}

\section{Petra Dannapfel $^{1 *}$, Eva Törnvall ${ }^{2}$ and Ewa Wressle ${ }^{3}$}

${ }^{1}$ Department of Medical and Health Sciences Social Medicine and Public Health Linköping University

${ }^{2}$ Management Department, Region of Östergötland, and Department of Medical and Health Sciences, Linköping University, Linköping, Sweden

${ }^{3}$ Department of Acute Internal Medicine and Geriatrics, Linköping University, Linköping, Sweden

\begin{abstract}
Introduction: The aim of this study was to evaluate participants' and managers' experience of the design and content of an education programme. The Knowledge to Action (KTA) framework was applied to identify the steps of knowledge creation and action in the education programme.

Methods: Data were collected from 18 participants representing two groups: participants in the intervention and supervisors and managers. Two focus groups took place: two with participants in the intervention (4 and 3 in each) and one with eleven managers.

Results: All steps in the KTA framework were identified and discussed from several aspects. The importance of selecting projects that were relevant and added value in their clinics was mentioned by all participants. The participants also mentioned that after the education, they had further understanding and increased skills in how to be active and perform continuous improvement projects. The step in the KTA process regarding how to adapt knowledge to local context was not discussed explicitly by the participants or managers.

Discussion: Education in research methods and performing improvement projects to develop the clinic creates a more positive attitude to working with continuous improvement. The participant's self-esteem and knowledge increased regarding how to work with improvements. It is important to have the manager's support to perform a project. Emphasis was on knowledge inquiry and synthesis and presenting the results with or without possible solutions. The participants and managers talked about barriers and knowledge use more generally and at an organizational level. This means that the participants did not gain the last bit of knowledge needed to put the action into practice. This implies that the problem regarding lack of implementation skills in health care might remain.
\end{abstract}

Keywords: Continuous education; Clinicians; Knowledge to Action (KTA)

Abbreviations: EBP: Evidence-Based Practice; KTA: Knowledge to Action; PDSA; Plan, Do, Study, Act; RDU: Research and Development Unit

\section{Introduction}

Knowledge is constantly changing and a great deal of the knowledge that is learned during training at university is obsolete by the time of graduation [1]. In many areas, such as health care, up-to-date knowledge is critical, as is the ability to reflect and apply it in practice [2]. Several challenges related to translating new research into changes in the care given to the patient have been explored in earlier studies [3-5]. Organizations need to make sure that professionals have time and access to continuous education and development. Peck et al. [6] define "continuing professional development" as the process by which health professionals' keep updated to meet the needs of patients, the health service, and their own professional development. It includes the continuous acquisition of new knowledge, skills, and attitudes to enable competent practice."

1. If clinicians are to deliver care based on the best evidence available, they need to access, appraise and integrate research literature into their decision making. However, that requires professionals to have skills in searching for, synthesizing and applying evidence in a way that fits the clients [7]. EvidenceBased Practice (EBP) was originally regarded as a problemsolving process, and sometimes referred to as a decisionmaking or a critical appraisal process comprising five steps to be undertaken by the practitioner when faced with clinical uncertainty:
2. Formulating an answerable question based on a patient's problems.

3. Seeking out the best relevant evidence.

4. Critically appraising the validity and usefulness of this evidence.

5. Integrating this appraisal into practice and patient preferences.

6. Assessing the results [8].

There are several reasons why new knowledge or improvements are not implemented; e.g. the environments and settings in which the knowledge is to be implemented are not properly designed for this purpose; withholding barriers that need to be addressed. However, a lack of knowledge about research methods, critical appraisal of research results and lack of time and facilitation from leaders may also be hindrances [5]. To develop clinicians' competencies regarding the prerequisites needed for EBP skills include access to evidence, critical appraisal and structured support to implement and evaluate evidence $[7,9]$. Many of the individual barriers to implementation of EBP e.g. lack of time to search for and appraise research and insufficient support

*Corresponding author: Petra Dannapfel, PhD, Karolinska University Hospital Stockholm, Sweden, Tel: 0727174370; E-mail: petra.dannapfel@sll.se

Received August 02, 2017; Accepted August 26, 2017; Published August 29, 2017

Citation: Dannapfel P, Törnvall E, Wressle E (2017) Education to Increase Skills in Research Methods among Clinicians in Health Care. J Health Med Informat 8: 282 doi: 10.4172/2157-7420.1000282

Copyright: ( 2017 Dannapfel P, et al. This is an open-access article distributed under the terms of the Creative Commons Attribution License, which permits unrestricted use, distribution, and reproduction in any medium, provided the original author and source are credited. 
for this process, have been linked to organizational issues, suggesting that organizational culture is an important inhibiting factor to implementing EBP [10-12].

Top management including managers and directors for primary health care in the Östergötland County Council identified that research activity and research skills among staff were low. Therefore, a decision was made to invest in training in research methods to facilitate continuous improvement in the clinics. The purpose of the education programme was to introduce, generate awareness and enhance clinicians' ability to critically appraise and integrate new research evidence into practice. Expectations were that better skills and confidence in research methods would increase the integration of research evidence into practice. Further, these skills would foster new ways of thinking regarding being critical, undertaking continuous improvement and keeping oneself up to date. The Research and Development Unit (RDU) was assigned to develop an educational programme with the aim of increasing clinicians' skills in research methods with a focus on patient safety.

The aim of this study was to evaluate the educational intervention in terms of participants' and managers' experience of the design and content of the programme. The Knowledge to Action framework by Graham et al. [13] was applied to identify the steps of knowledge creation and action in the education programme.

\section{Theoretical framework}

Knowledge to Action (KTA) is a conceptual framework that can be divided into two concepts: knowledge creation and action. However, in reality, the process of creation and application of knowledge is far more dynamic. Graham et al. [13] emphasize three aspects that need to be understood for continuing education in the health professions:

1. The complete KTA process.

2. The range of stakeholders involved beyond practitioners.

3. Conceptual frameworks that may be useful and facilitate the integration of research into practice.

The KTA assumes a systems perspective where producers and users of knowledge are situated in a social system. The system is both adaptive and responsive and the KTA process must be dynamic and iterative regarding the creation and application of knowledge, and the seven action phases may take place at the same time or sequentially [14].

\section{Methods}

\section{Study setting}

The provision of health care services in Sweden is primarily the responsibility of the 21 county councils throughout Sweden [15]. Every year, an employee survey is sent to everyone in the county council, which includes items on the use of research results and involvement in research. For Östergötland County Council, this survey indicated the need for development in both areas which generated a discussion by top management about a suitable intervention for improvement. The discussion lasted for a year and involved discussion with the RDU.

The intention with the education was to find a common activity to increase knowledge regarding research methods and for clinicians to acquire advanced skills in research and development methodology to more actively participate in or conduct their own improvement projects within the clinic.

\section{Design}

Description of the intervention: The education was a 1-year programme and included five full days with lectures and one examination day. The course includes lectures concerning qualitative and quantitative methods, ethics, the plan, do, study, act (PDSA) cycle, and how to write a project plan and perform a project. Each student was allocated one or two tutors from the RDU. Between the educational days, the participants worked with individual projects with support from the tutors. The participants were also offered group and/or individual support with their examination task. The date and time for these meetings were scheduled from the start. The project was part of the examination, and to pass the course, the students had to investigate a real problem related to patient safety in their clinic using scientific methods. Participants were also asked to suggest actions to improve their identified problem area. On the examination day, participants presented their project both orally and with a poster. A plan for implementation of their results and how they could continue work towards improvements in their clinic were also included in the final project report.

Data were collected from 17 participants representing two groups: participants in the intervention and supervisors and tutors of the intervention and managers. During 2014, two focus groups took place in accordance with Barbour and Kitzinger [16]: Two with participants in the intervention ( 4 and 3 persons in each) and one with ten managers. Participants for the focus groups were recruited by ET and EW who was both also involved in creating the content of the intervention.

Participants: The objective was to recruit participants from all three groups (intervention participants, managers and tutors) to gain different perspectives regarding the intervention. The groups also represent different professions in the organization, thus enhancing the validity of the study.

Intervention participants: The recruitment of participants to the intervention was performed by the clinic managers and was different for each clinic, e.g. several clinic managers asked the middle managers to help find a suitable candidate. Some managers sent an email with information about the programme and an invitation to enrol other managers to hand picks participants.

Fifteen participants participated in the programme. One of the participants worked in primary care and 14 worked in hospital settings. There were seven nurses, five physicians, one physiotherapist, one occupational therapist and one social worker; two male and twelve females (age range 26-45). All participants were asked to take part in the study and seven accepted; two physicians, five females (age range 26-40). All fifteen participants completed the full program and finalized the exam.

Managers: All managers involved in making the decision that this intervention was to be prioritized were included in one focus group. There were six physicians, two physiotherapists and three with other professions; five male and six females (age range 40-64).

\section{Data collection}

The study applied an abductive approach using an interview guide containing open-ended questions but also questions related to the different steps in the KTA process. The guide was developed by the authors of the study. It was scrutinized in a seminar with representatives from the RDU; no major revisions in the interview guide were made afterwards. 
Each interview began with an open question asking the participants to describe their overall experience and content of the intervention programme. Experience of the intervention could include hindrances and facilitators for participation and the application of the new knowledge to praxis, and this in relation to working systematically with continuous improvement and knowledge use.

We considered use of new knowledge in a broad sense, encompassing both direct (instrumental) use in terms of changing clinical practice based on new knowledge, i.e. research- or experiencedbased knowledge or indirect (conceptual) use, meaning that new knowledge influenced the professionals thinking (understanding, knowledge and attitudes) concerning clinical practice issues [17].

The interviews were conducted during regular working hours to facilitate participation. Each focus group interview lasted between 40 and $60 \mathrm{~min}$. Informed consent was obtained by informing the participants that participation was confidential and voluntary and that they could withdraw at any time during the interview. Two moderators attended all focus groups. The first author (PD) of this study acted as moderator leading the interviews and asking follow-up questions. The second researcher $(\mathrm{SH})$ took field notes and made observations, however these observations were not analysed in this study and thus not part of the results. In general, discussions in the groups were fluent and little steering from the moderator was needed. All participants had gone through all steps in the intervention.

\section{Data analysis}

Interviews were recorded with a Dictaphone and transcribed verbatim by the first author. The data were analysed using qualitative content analysis, a technique for analysis of texts grounded in empirical data with an explorative and descriptive character [18]. As a first step, all authors read all transcripts to obtain an understanding of the whole. The transcripts were then coded by the first author using directed content analysis, which entails a structured analysis process to code and categorize the data [19]. The next step in the process was to highlight words in the text that captured various key statements and thoughts in relation to the KTA framework [19]. The researchers approached the text several times. During this process, codes that reflected more than one key statement or thought developed; the codes were then aggregated into clusters based on similarity of the content and their relation to each other and the KTA framework steps [19]. After reexamination, the initial clusters were merged into categories.

In the next step, the findings concerning the content of the categories were compared with the KTA framework using a deductive approach. The categories were then mapped onto the different steps in the theory, as described in the next section. Discussion on this mapping process continued until no inconsistencies existed and a shared understanding was reached to prevent researcher bias and strengthen the internal validity [20].

The categories were cross-examined to ascertain that they were defined in such a way that they were internally as homogeneous as possible and externally as heterogeneous as possible and described a specific step in the KTA process [18]. All authors discussed the contents of the categories using triangulating analysis, i.e. the authors independently analysed the same data and compared their findings.

Representative quotations were identified to report the findings. Quotations were then translated from Swedish to English. The first author (PD) and author (EW) translated the quotes from Swedish to English.

\section{Results}

\section{Identify problem}

The first step in the KTA process is to identify the problem or issue that needs improvement. This first step in this study is to acknowledge the need to improve skills to work more systematically with continuous improvement at the overall organizational level. At the clinical level, the first step was to identify a specific area for improvement for the individual projects. During discussions in the top management group, it came clear how differently the clinics worked with research and development and that many employees lacked skills in research methods. "We don't use research to the extent we should in our centre so this was a way to increase the awareness and the presence of research and development more generally in our centre" (manager, 1 NSC). "There are several aims with R\&D at different levels and this is about the individual perspective on $\mathrm{R} \& \mathrm{D}$ but also to work towards a common perspective" (manager, 5 NSC). The managers mentioned that increasing the overall knowledge regarding research and development is part of their strategy to have a more research aware staff and researchers in their clinics. "We have an overall need to develop R\&D" (manager, 2 NSC). Further, the managers believe that having employees who perform improvement projects "can inspire and raise the interest, thus some employees become ambassadors for R\&D" (manager 1, NSÖ).

The decision to implement the 1-year programme with a focus on patient safety was sanctioned by the Director for the Center of Local Care: "It was a long journey and a lot of discussions during one year regarding economy and taking employees out of patient care and production; however, during this journey everyone changed their minds; if we are to provide safe care, we also need to conduct research on how and what to do" (manager 3, NSC).

\section{Review and select knowledge}

One topic mentioned in the aim of the intervention and by all respondents was the importance of selecting a relevant project that added value in their clinics. The scope of the project had to be derived from real practice, reviewed and found important for the clinic. The participants who were chosen to participate in the education programme all had influence over the content of their projects. However, project ideas were discussed with the managers at the clinics and joint decisions were made on the project content. "I immediately got an idea, after that I discussed with my manager if that was what the clinic needed" (participant 1, focus group 1). My manager was happy that I wanted to participate, "do what you like; we need improvement in so many areas" (participant 3 , focus group 1). All participants agreed that the project had to have its origin in a real problem that needed to be improved in the clinic; it is important that it is relevant to practice. This was also highlighted by the managers who also stated that patient safety is a broad topic that permeates almost everything in the clinic.

\section{Assess barriers to knowledge use}

Most of the participants experienced a positive attitude from their managers regarding participation in the education. They discuss the importance of support from the managers so that they were given time to work with their projects. Managers have a crucial role in creating a culture whereby improvement initiatives are integrated in practice. "Our manager is positive to all research and development initiatives and very willing to give us time for that. I have heard that it is not always the case. I think that it depends on what kind of manager you have" (participant 3, focus group 1). Even though the managers were 
generous with time, the participants expressed that they still had too much clinical work to do: "Yes take the time you need is the saying from the managers, however that is not so easy when you stand in the clinic with all your tasks to perform and no one else to help" (participant 2, focus group 1). This dilemma was also mentioned by the managers: "this becomes an issue, first and foremost on a clinical level, but also for the individual who is stuck in a difficult situation about how to prioritize" (manager 1, NSÖ).

The participant believed that managers with more research experience are more willing to facilitate conducting improvement projects more systematically in the clinic. "If the manager has research experience, I believe that he or she has another focus and understands that this is systematic work ... a leader can have many good qualities but I think that this might be crucial" (participant 2, focus group 1). "Maybe our managers need some kind of update to have a more scientific approach, then they can start to implement a more scientific and evidence-based work place" (participant 3, focus group 2).

Involvement in the intervention means absence from work with patients. For the participants, it was important that their colleagues understood their absence from clinical work. The managers play an important role in this. "We need support from management and colleagues that it is okay, that they understand that this will be useful for our practice and that we cannot give a 100\% during this time in the daily work with patients" (participant 1, focus group 2). The participants believe that their colleagues were a bit ambivalent. Their colleagues believed it was important, but at the same time they are left with the clinical work and an increased work load. One participant made comments such as "okay you will be gone again, for how long are you going to work with your project?" (Participant 4 , focus group 1) But at the same time they are curious and supportive and ask questions about the progress of the project. "It is stimulating (the project) and you want to infect your colleagues with this energy" (participant 1 , focus group 2). Some colleagues have also been involved in projects, e.g. filled in questionnaires. One participant mentioned that her colleagues did not notice her involvement in the education programme because she worked on her project during weekends and evenings.

\section{Select, tailor and implement interventions}

Time is mentioned by the managers as critical. The respondents' participation in the education programme takes time from production. This is also the case with an employee participating in education. "I believe that it is important to estimate how much time it will take to perform the education programme and the project; dialogue regarding resources is crucial" (manager 2, NSÖ). On the other hand, the managers mentioned that continuous improvement and development are to be integrated in daily practice "to be able to give the patient better care and work smarter; this should also be notable in the economy. At the same time the pressure in the wards is high and we lack personnel" (manager 1, NSV).

Results from the projects are to be implemented in the clinics. However, the participants mentioned that they lack knowledge regarding implementation: "the aim is to implement my suggestions for improvement. I believe that implementation is a long and demanding process and we lack knowledge regarding how to perform an implementation" (participant 2, focus group 1).

\section{Continuous use of new knowledge}

All participants mentioned that they have started to think "scientifically". One example is to measure changes both before and after implementation to recognize if there have been any improvements.
The participants mentioned that continuous discussions about research at the workplace are important if learning and development initiatives are to be integrated in practice. "We have no natural forum in which we can highlight these issues. The meetings we have are filled with other things we need to act on, the most acute lack of personnel, how do we solve that, you need time to follow up on your ideas or projects ... it is about expectations on us. Here we are to work with evidence and nothing else, it's not up to you to decide if you are to work in accordance with evidence" (participant 3 , focus group 1).

Some participants emphasized that all small changes are vital. If practice is to change, it is not the bigger change efforts but rather small and continuous improvements that will make practice better. "As soon as you talk about improvements, it feels like you are to make over the whole hospital; however, small improvements that can change practice from the morning till the evening also matters" (participant, 3 focus group 2).

Participants emphasized that management must ask for this kind of project and improvement efforts. "I don't think that we talk about making improvements or use evidence from research in comparisons with how much things cost; research is sadly just a tiny part. For example, it says production plan, already here it is wrong. It should say quality plan or similar, so you start to think from that perspective; the focus should be clear and help us to lead more improvement and development changes" (participant 1, focus group 2). "Top management can be sharper and clear about how we participate in development, and also follow up on activities that we have done in this area" (manager 10, NSÖ).

The managers mentioned that work with improvements often remains in the background behind the requirements of production. Availability and budget are prioritized. "It is clear that the number in the bottom line is what counts" (manager 4, NSV). One manager mentioned that if they do not work with development and improvement "we will slowly cut the branch that we are sitting on and the patient will not receive the best care" (manager, 3 NSV). The participants and managers all mentioned that development and improvement are about creating a culture with scientific thinking. This should be done in cooperation with all clinics and be highlighted in the overall organization.

The step in the KTA process regarding how to adapt knowledge to the local context was not discussed explicitly by the participants or managers. Discussions regarding adaption take place when planning a project so that it is close to practice and the content of the project is derived from a real problem. Adaption to local context is mentioned but just briefly and because the project investigated a local problem, the fit is in a way inherent in the solution.

\section{Discussion}

The aim of this study was to evaluate the educational intervention in terms of participants' and managers' experience of the design and content of the programme and execution of the intervention. The KTA framework by Graham et al. [13] was applied to identify the steps of knowledge creation and action in the education programme.

The professional's in the RDU designed the education programme. The programme mostly concerned the creation of knowledge and less emphasize was placed on the action phase/part as described in the KTA framework. In the programme, the participant's task was to investigate a real problem in their clinic. It is likely that the participants are more motivated to perform the project if it is derived from an 
existing knowledge inquiry within their clinic. Investigation of a local problem might also increase understanding of how improvement can be performed continuously in their clinic with the methods learned in the programme. Further, adult students are task- or problem centred when it comes to learning [19-21]. After identifying a problem, they performed the review and select knowledge steps in the KTA framework. The participant was not obliged to come up with a solution in the framework of the programme.

The steps in the programme related to investigation of the potential fit of the actions and implementation, which can be compared with the KTA steps, adapt to local context, assess barriers and tailor implementation, were not fully applied. These steps are mentioned in the programme but not applied in practice; no tutorial in the programme discussed these steps and only a few of the clinicians mentioned this specifically. Emphasis was rather on knowledge inquiry and synthesis and presenting the results of their project with or without possible solutions. The participants and managers talked about barriers and knowledge use more generally and at an organizational level. This means that the participants still did not gain the last bit of knowledge needed to put the action into practice. This implies that the problem regarding lack of implementation skills in health care might remain.

When planning for the intervention, the professionals at the RDU lobbied for the urgency of this matter among managers to gain support. Earlier research shows the importance of manager support to facilitate allocation of the time and resources needed to be involved in further education and work with their project. The top management all agreed that this programme was to be prioritized. In health care, there is often a tension between production (treating patients) and work with improvements i.e. projects aimed to improve how the work is performed. Time and resources were also a challenge for the current intervention. Despite consensus among managers to let the clinicians participate during work hours, the clinicians spent quite some time on the project after work because of the high work load with patients in the clinic. The clinicians felt obliged to help their colleagues; the project became secondary when the clinic lacked personnel. Lack of time is a barrier for further education, as is difficulty in balancing between work and educational demands $[22,23]$. However, some of the clinician's mentioned that their colleagues think they spend too much time on their project, indicating that not all stakeholders support the programme.

The third aspect mentioned in KTA framework, conceptual frameworks that may be useful and facilitate the integration of research in practice, has yes and no answers. The yes answer is the inspiration of the KTA framework when planning the intervention, although the product had limited fidelity to all parts of KTA framework. The training in the knowledge creation steps is essential to perform any implementation; no object to implement, no implementation. The first steps in KTA are to identify a problem, review and select knowledge. This was all included in the programme through tutorials, and workshops in the library improved database search skills. In the programme, the participants were taught the PDSA as a guiding model. Several participants highlighted this as a useful tool to guide how to perform any improvement. The intervention involved both instrumental and conceptual learning, both involving concrete application and changes in knowledge and understanding [17]. Learning a useful model relates to the KTA step about sustaining knowledge because this can help clinicians to keep updated. The PDSA cycle includes steps on what to improve and to evaluate outcomes, both of which are included in the KTA framework. In the tutorials and discussions, the intervention had little focus on how to utilize, disseminate or implement knowledge, which includes four steps in the KTA, mentioned above: adapt knowledge to local context, assess barriers to knowledge use (this step is related to structure and the culture that exists in the clinic and more a question for the manager and the work group including all stakeholders). Further, the steps on selecting, tailoring and implementing interventions are mentioned in the programme as important, but not performed. However, some clinicians have plans on how to implement an intervention based on the results from their project. The step on monitoring knowledge used was not mentioned or discussed.

The results show that education in research methods and performing improvement projects that are useful in developing the clinic create a more positive attitude to working with continuous improvement. Further, the participant's self-esteem and knowledge increased regarding how to work with improvements. The importance of having the manager's support to perform the project was highlighted by all the focus groups. To create a culture that facilitates a learning milieu, all actors at all levels in the organization need to work together. It is not only about time and resources; it is also about an innovative climate that embraces new way of thinking and acting.

\section{Lessons learned}

The evaluation from this first education intervention has become the basis for subsequent education interventions. In the following years, the act of adapting project work at each work place will, involve discussions between the management, the participant and the tutor about a realistic extent of the project. Further, it seems to be an advantage to have two participants per clinic, from different professions, work on the same project. This provides strength to carry on and leads understanding over the professional barriers. Several physicians participated during their specialization studies. Still, there is a need for more focus on implementation and probably that need its own intervention. Successful implementation needs more strategies than an educational intervention [24].

\section{Competing Interests}

The authors declare that they have no competing interests.

\section{Authors' Contributions}

All authors contributed actively to this paper. PD wrote the first draft, which were discussed EW and ET. Further drafts were developed in close collaboration among all three authors. All authors approved the final version of the paper.

\section{References}

1. Grol R, Wensing M (2005) Improving patient care: the implementation og change in clinical practice. Qual Saf Health Care 15: 447

2. Berggren E, Orrevall $Y$, Ödlund Olin A, Strang P, Szulkin R, et al. (2015) Evaluation of a continuing educational intervention for primary health care professionals about nutritional care of patients at home. The Journal of Nutrition, Health \& Aging 20: 428-438.

3. Grimshaw J, Eccles M, Thomas R, MacLennan G, Ramsay C et al. (2006) Toward evidence-based quality improvement. Evidence (and its limitations) of the effectiveness of guideline dissemination and implementation strategies 1966-1998. J Gen Intern Med 21: S14-S20.

4. Grol R, Grimshaw J (2003) From best evidence to best practice: effective implementation of change in patients' care. Lancet 362: 1225-1230.

5. Dannapfel P, Peolsson A, Nilsen P (2013) What supports physiotherapists' use of research in clinical practice? A qualitative study in Sweden. Implement Sci 8: 31 .

6. Peck C, McCall M, McLaren B, Rotem T (2000) Continuing medical education and continuing professional development: international comparisons. BMJ 320 432-435. 
Citation: Dannapfel P, Törnvall E, Wressle E (2017) Education to Increase Skills in Research Methods among Clinicians in Health Care. J Health Med Informat 8: 282. doi: 10.4172/2157-7420.1000282

Page 6 of 6

7. Glegg S, Livingstone R, Montgomery I (2015) Facilitating interprofessional evidence-based practice in paediatric rehabilitation: development, implementation and evaluation of an online toolkit for health professionals. Disabil Rehabil 38: 391-399.

8. Sackett DL, Rosenberg WM, Gray JA, Haynes RB, Richardson WS (1996) Evidence-based medicine: what it is and what it isn't. BMJ 312: 71.

9. Fineout-Overholt E, Melnyk, B (2005) Building a culture of best practice. Nurse Lead 3: 26-30.

10. Nutley SM, Walter I, Davies HTO (2007) Using evidence: how research can inform public services. Bristol 86: 618-618.

11. Sibbald SL, Wathen CN, Kothari A (2016) An empirically based model for knowledge management in health care organizations. Health Care Manage Rev 41:64-74.

12. Williams B, Perillo S, Brown T (2015) What are the factors of organisational culture in health care settings that act as barriers to the implementation of evidence-based practice? A scoping review. Nurse Educ Tod 35: 34-41.

13. Graham ID, Logan J, Harrison MB, Straus SE, Tetroe J, et al. (2006) Lost in knowledge translation: time for a map? Contin Educ Health Prof 26: 13-24.

14. Graham ID, Tetroe J (2007) Some theoretical underpinnings of knowledge translation. Acad Emerg Med 14: 936-941.

15. http://www.regeringen.se/sa-styrs-sverige/
16. Barbour RS, Kitzinger J (1999) Developing focus group research, politics, theory and practice. Thousand Oaks, CA: Sage Publications.

17. Estabrooks CA (1999) Will evidence-based nursing practice make practice perfect? Can J Nurs Res 30: 15-36.

18. Krippendorff K (2004) Content analysis. An introduction to its methodology. Org Res Met 13: 392-394.

19. Hsieh HF, Shannon SE (2005) Three approaches to qualitative content analysis. Qual Health Res 15: 1277-1288.

20. Patton M (2000) Qualitative evaluation and research methods. London: Sage.

21. Bengtsson M, Ohlsson Bodil (2010) The nursing and medical students motivation to attain knowledge. Nurse Educ Today 30: 150-156.

22. Altin SV, Tebest R, Kautz-Freimuth S, Redaelli M, Stock S (2014) Barriers in the implementation of interprofessional continuing education programs-a qualitative study from Germany. BMC Med Educ 14: 227.

23. Baker R, Camosso-Stefinovic J, Gillies C, Shaw E, Cheater F, et al. (2010) Tailored interventions to overcome identified barriers to change: effects on professional practice and health care outcomes. Cochrane Database Syst Rev 3: CD005470.

24. Dogherty EJ, Harrison MB, Graham ID, Vandyk AD, Keeping-Burke L (2013) Turning knowledge into action at the point-of-care: the collective experience of nurses facilitating the implementation of evidence-based practice. Worldviews Evid Based Nurs 10: 129-139. 